\title{
PREVISÃO DE CHUVA A CURTÍSSIMO PRAZO NA ÁREA DE ABRANGÊNCIA DO RADAR METEOROLÓGICO DE SÃO PAULO
}

\author{
JOSÉ FELIPE DA SILVA FARIAS ${ }^{1}$, AUGUSTO JOSÉ PEREIRA FILHO² \\ ${ }^{1}$ Instituto Nacional de Pesquisas Espaciais, Centro de Previsão do Tempo e Estudos Climáticos (INPE/CPTEC), \\ Cachoeira Paulista, SP, Brasil \\ ${ }^{2}$ Universidade de São Paulo (USP), São Paulo, SP, Brasil, \\ jose.farias@cptec.inpe.br, apereira@model.iag.usp.br \\ Recebido Março de 2012 - Aceito Setembro de 2012
}

\begin{abstract}
RESUMO
A avaliação da previsão de chuva a curtíssimo prazo com até 3 horas de antecedência na área de cobertura do Radar Meteorológico de São Paulo (RSP) para diferentes tipos de sistemas precipitantes, principalmente os associados às enchentes e deslizamentos na Região Metropolitana de São Paulo (RMSP), foi realizada por meio de um modelo advectivo a partir do campo de vento 2D médio e da velocidade dos campos das taxas de precipitação estimados com o radar e um Esquema Numérico de Terceira-ordem Corrente Acima (ENTOCA), que utiliza um vetor de deslocamento constante. O desempenho obtido para precipitação acumulada foi avaliado com os seguintes parâmetros estatísticos: Índice de Sucesso Crítico (ISC), Probabilidade de Detecção (POD), Razão de Falsos Alarmes (RFA), Erro Quadrático Médio (EQM) e Coeficiente de Correlação (CCOR). O ISC para limiar de acumulo de $0,2 \mathrm{~mm}$, em períodos de acumulação de 60 minutos, apresentou os seguintes valores: $77 \%$ para eventos de Frente fria, 67,5\% para Linhas de Instabilidade, 58\% para Bandas Dispersas, 56,4\% para Convecção Isolada e $47 \%$ para Brisa Marítima. A previsibilidade obtida foi maior para sistemas estratiformes do que para sistemas convectivos. A partir de períodos de 90 (120) minutos de advecção o CSI diminui exponencialmente para sistemas convectivos (estratiformes).
\end{abstract}

Palavras chave: Enchentes, Hidrometeorologia, Previsão de chuva a curtíssimo prazo.

\begin{abstract}
RAINFALL SHORT-TERM FORECAST IN THE SURVEILLANCE AREA OF SÃO PAULO WEATHER RADAR

The evaluation of the rainfall nowcasting within the surveillance area of São Paulo weather radar for different types of precipitating systems, mainly those associated to floods and landslides in Metropolitan Area of São Paulo, was carried out with a 2D wind advective scheme and rainfall rates estimated by radar. The third-order upstream numerical scheme was used with an uniform wind vector. The rainfall forecast skill for a given time interval was evaluated using the Critical Success Index (CSI), Probability of Detention and False Alarm Rate statistic parameters. Quantitatively, the accuracy of the forecast was evaluated with the mean-square error. The correlation coefficient showed better skill for stratified precipitations than for convective precipitations. The average skills based on critical Success Index for the thresholds of $0.2 \mathrm{~mm}$ at 60 minutes of accumulated precipitation are: Cold fronts (77\%), Squall lines (67,5\%), Dispersed Bands (58\%), Isolated Convection (56,4\%) and Maritime Breeze (47\%). In general, from 90 minutes of advection (convective systems) to 120 minutes (stratiform systems), the forecast skill decreases.
\end{abstract}

Keywords: Flash flooding, Hydrometeorology, Rainfall nowcasting. 


\section{INTRODUÇ̃̃̃O}

O aumento nos últimos anos da disponibilidade de dados de radar com alta resolução espacial e temporal, desenvolvimento de técnicas de automatização computacional e de novos métodos para aplicação na previsão de chuva a curtíssimo prazo (0-3h) (Einfalt et al. 1990) tem possibilitado a análise de eventos de precipitação intensa com algumas horas de antecedência, principalmente em regiões de adensamento populacional, como é o caso da Região Metropolitana de São Paulo (RMSP).

O radar meteorológico é um equipamento de sensoriamento remoto ativo da atmosfera adequado para monitorar os sistemas precipitantes, devido à sua alta resolução espacial e temporal (Wilson et al. 1998). Entretanto, a necessidade de uma quantificação da acurácia da estrutura da distribuição espacial e da variabilidade temporal da velocidade e precipitação dificultam a utilização direta dos dados de radar para a previsão de chuva a curto prazo. A previsão da quantidade de chuva numa determinada área inclui incertezas, e testes com modelos hidrológicos mostram que o erro em quantificar a precipitação é responsável pelos erros encontrados nas previsões hidrológicas (Novak, 2007). Esses erros também variam de acordo com o tipo de sistema precipitante avaliado, bem como de acordo com sua estrutura e grau de organização, conforme estudo de Pereira Filho et al.(1999).

A quantificação da precipitação em um determinado local, um dos fatores que limita a acurácia da previsão de chuva a curtíssimo prazo, foi considerada por diferentes pesquisadores. Por exemplo, Johnson e Brás (1980) desenvolveram procedimento estatístico com base em observações de pluviômetros. Georgakakos e Brás (1984a,b) utilizaram observações meteorológicas de superfície e pluviômetros. Browning e Collier (1989) revisaram uma ampla classe de processos, associados à nowcasting, e entendida neste trabalho como "previsão de eventos repentinos". Tais processos são amplamente aplicados operacionalmente (Elvander, 1976; Bellon e Austin, 1978, 1984; Browning et al. 1982; Walton e Johnson, 1986; Einfalt et al. 1990). Entre as ferramentas de nowcasting, a precipitação pode ser extrapolada por advecção, com uso da distribuição do vetor velocidade do vento estimado da análise do vetor deslocamento do sistema precipitante a partir das imagens mais recente do radar. Entre as vantagens do método advectivo têm-se:

1. automatizar a previsão da precipitação,

2. apresentar resolução temporal e espacial compatível com a resolução dos dados de radar e,

3. ser menos sensível às mudanças morfológicas dos sistemas.
Além disso, o método mostra trajetórias mais realísticas dos sistemas precipitantes observados pelo radar, quando o escoamento médio da troposfera conduz de forma definida o sistema precipitante.

O método advectivo também apresenta algumas desvantagens. Por exemplo,

1. carregam consigo os erros de estimativa do campo de precipitação do radar, mesmo se a equação do radar (Bringi e Chandrasekar, 2005), tenha sido calibrada previamente,

2. não consideram explicitamente a dinâmica dos sistemas convectivos (Gao e Li, 2010) e

3. erros causados pela diferença da escala de tempo do fenômeno e do escoamento advectivo.

Formalmente, o período de advecção não pode ser maior do que uma fração de escala de tempo do ciclo de desenvolvimento da tempestade ou do sistema convectivo precipitante. Desde que a escala de tempo de células convectivas individuais da tempestade é de aproximadamente 20 minutos, a etapa de advecção entre a localização inicial e final da célula convectiva não deve ser este período. Já os sistemas definidos por agrupamento de tempestades, nos sistemas convectivos e estratiformes apresentam escalas de tempo maiores, o que permite estender o período de advecção por algumas horas.

Neste trabalho, o banco de dados de radar para as taxas de precipitação tem frequência de amostragem de 5 minutos. Verificou-se que em amostragens de até 30 minutos não ocorre perda significativa da resolução espacial dos sistemas precipitantes observados. Assim, para optimização da demanda computacional, empregou-se passos de tempo de 30 minutos entre as etapas sucessivas de advecção.

Saliente-se que, como nota histórica, os primeiros resultados baseados em técnicas de extrapolação de ecos de radar foram obtidos por Austin e Bellon (1974), sendo que desde então são amplamente utilizados, principalmente para previsão de chuva em bacias urbanas.

Em geral, compara-se o desempenho do nowcasting com o desempenho de previsão numérica com modelos de mesoescala. Para um intervalo de tempo curto (de até $3 \mathrm{~h}$ ), o nowcasting baseado em advecção lagrangiana apresenta um desempenho melhor do que os modelos de previsão para a taxa de precipitação, enquanto que para períodos maiores (acima de $6 \mathrm{~h}$ ), os modelos de previsão apresentam melhor desempenho do que o nowcasting. Isto está associado ao período de spin-up necessário para o ajuste interno dos modelos de previsão em mesoescala, o que atrasa o início da precipitação. Já o nowcasting inicia-se diretamente com a condição inicial do sistema precipitante, degradando a qualidade de sua previsão com o passar do tempo, uma vez que tende a conservar a estrutura dos sistemas precipitantes, não considerando explicitamente sua evolução. 
De acordo com Sálek et al. (2006), resultados de testes com modelos hidrológicos utilizando extrapolação de ecos de radar mostram que é possível prever enchentes com minutos de antecedência, principalmente em bacias pequenas. A previsão de chuva a curtíssimo prazo é de suma importância principalmente em áreas muito habitadas, auxiliando na redução das fatalidades causadas por tornados, tempestades e enchentes repentinas (flash flooding), além de evitar grandes prejuízos aos diversos setores da sociedade como o privado, industrial, transporte e agrícola.

O objetivo deste trabalho foi verificar previsões de chuva a curtíssimo prazo (0-3h) com o radar meteorológico de São Paulo (RSP), para diferentes sistemas precipitantes, por meio de um modelo advectivo que utiliza o campo do vento 2D médio, a velocidade de deslocamento dos sistemas precipitantes e um Esquema Numérico de Terceira Ordem Corrente Acima, denominado ENTOCA (Austin e Bellon, 1974; Tremback et al., 1987; Pereira Filho et al., 1999). A seção 2 apresenta a metodologia utilizada nesta pesquisa para a obtenção da previsão de chuva em curto prazo com dados do radar de São Paulo entre 2003 e 2007, assim como, a estatística da avaliação da previsão de chuva. A seção 3 apresenta os resultados e discussões e, por fim, na seção 4 são apresentadas as conclusões.

\section{DADOS E METODOLOGIA}

\subsection{Previsão de chuva a curtíssimo prazo}

A previsão de chuva a curtíssimo prazo pode ser realizada a partir da determinação do vetor deslocamento do sistema, por exemplo, obtido pelo método SHARP (Short-term Automated Radar Prediction) proposto originalmente por Austin e Bellon (1974). Neste método, o campo de precipitação atual é advectado (isto é, transladado, rotacionado e deformado) dentro da área de cobertura do radar, desde sua origem até um determinado destino de previsão de acordo com o vetor deslocamento associado.

\subsection{Obtenção do Vetor deslocamento do sistema pelo método SHARP}

Neste trabalho, emprega-se o método SHARP, desenvolvido pelo grupo de meteorologia da McGILL University, aqui usado para extrapolação linear dos ecos do RSP, para até 3 horas de precipitação acumulada. Supõe-se que o sistema precipitante, uma vez identificado na área de cobertura do radar, possa ser deslocado no tempo com vetor deslocamento constante. Portanto, o sistema mantém a mesma estrutura ao longo do deslocamento, sem modificação das suas áreas e taxas de precipitação. $\mathrm{O}$ algoritmo de previsão do radar RSP consiste de: a) cálculo do vetor deslocamento do sistema precipitante observado como um todo na área de cobertura do radar, determinado pelo coeficiente de correlação cruzada ( $\rho$ máximo) entre dois CAPPIs de altitude baixa separados por um intervalo de tempo de 30 minutos, e

b) previsão das taxas de precipitação a cada 5 minutos por até 3 horas, em pontos específicos dentro da área de cobertura do radar.

\subsection{Modelo advectivo de chuva}

O modelo advectivo foi desenvolvido para produzir previsão de chuva acumulada a curtíssimo prazo, baseado no deslocamento médio dos sistemas que utiliza correlações espaços-temporais dos dados de chuva. Esta técnica pode ser generalizada a partir da equação da continuidade 2D:

$$
\frac{\partial \varphi}{\partial t}+u \frac{\partial \varphi}{\partial x}+v \frac{\partial \varphi}{\partial y}=0
$$

sendo $\varphi$ o campo escalar, aqui, a taxa de precipitação em (mm $\left.\mathrm{h}^{-1}\right)$ e $(\mathrm{u}, \mathrm{v})$ as componentes horizontais do vetor velocidade do vento $\left(\mathrm{m} \mathrm{s}^{-1}\right)$. A solução da Equação 1 para $\varphi$ é obtida por um Esquema Numérico de Terceira-Ordem Corrente Acima, denominado ENTOCA (Pereira Filho et al., 1999, Pereira Filho, 2002), a partir da proposição original de Tremback et al. (1987).

A aplicação do modelo advectivo é baseada na consideração de que os ecos de chuva são aproximadamente constantes enquanto são advectados pelo vetor deslocamento (Pereira Filho, 1989). A aplicação do modelo foi realizada enquanto o sistema encontrava-se na área de cobertura do radar. O intervalo de 30 minutos entre o inicio de cada previsão foi utilizado para minimizar a má representação da dinâmica dos sistemas ao longo do deslocamento, evitando-se que eventuais novos núcleos de precipitação que surgissem na área do radar atingissem seu estágio maduro dentro do período de advecção. $\mathrm{O}$ vetor deslocamento obtido pelo RSP foi inserido no modelo advectivo e os sistemas advectados para os mesmos intervalos de tempo utilizados na acumulação, com o objetivo de comparar o campo de precipitação acumulado pelo radar e o campo obtido pelo modelo advectivo. Dessa forma, pôde-se verificar o desempenho das previsões para os diferentes tipos de sistemas precipitantes.

\subsection{Avaliação da previsão de chuva}

A avaliação da previsão foi realizada por meio do Índice de Sucesso Crítico (ISC), que é uma ferramenta estatística frequentemente utilizada para avaliar a previsão de variáveis meteorológicas. Assim, a precipitação acumulada pelo radar 
num determinado intervalo de tempo foi comparada com a precipitação acumulada prevista por meio de uma tabela de contingência para limites de acumulação pré-definidos. O desempenho das previsões foi também avaliado pela Probabilidade de Detecção (POD) e Razão de Falsos Alarmes (FAR) (Wilks, 1995). As seguintes expressões foram usadas para obtenção dos índices CSI, POD e FAR:

$$
\begin{aligned}
& C S I=\frac{\text { acertos }}{\text { acertos }+ \text { erros }+ \text { falsos alarmes }} \\
& P O D=\frac{\text { acertos }}{\text { acertos }+ \text { erros }} \\
& F A R=\frac{\text { falsos alarmes }}{\text { falsos alarmes }+ \text { erros }}
\end{aligned}
$$

Os índices CSI, POD e FAR foram classificados de acordo com o tipo de evento e resoluções espaciais da previsão. A Tabela 1 apresenta a contagem das previsões de curtíssimo prazo com resolução de $2 \mathrm{~km}$ para um determinado limite de chuva horária $(\mathrm{Po})$ contra os respectivos valores observados com o RSP e fórmulas de obtenção dos índices CSI, POD e FAR. Os valores de Po para este estudo foram de 0,2; 4,0; e $16,0 \mathrm{~mm}$.

A perda da acurácia das previsões no decorrer do tempo para cada tipo de sistema foi avaliada pelo coeficiente de correlação linear. Os erros das previsões em relação às observações com o RSP, para até $3 \mathrm{~h}$ de precipitação acumulada, foram analisados por meio do Erro Quadrático Médio (EQM), que pode ser escrito conforme Takacs et al. (1985).

$$
E Q M=E Q M_{a}+E Q M_{f}
$$

sendo $\mathrm{EQM}_{\mathrm{a}} \mathrm{o}$ erro de amplitude prevista em relação à observada e $\mathrm{EQM}_{\mathrm{f}} \mathrm{o}$ erro da fase prevista em relação à observada. $\mathrm{Na}$ forma matemática,

$$
\begin{aligned}
& E Q M_{a}=\left[\sigma\left(P_{o}\right)-\sigma\left(P_{p}\right)\right]^{2}+\left[\left\langle P_{0}\right\rangle-\left\langle P_{p}\right\rangle\right]^{2} \\
& \quad \mathrm{e} \\
& E Q M_{f}=2(1-\rho) \sigma\left(P_{0}\right) \sigma\left(P_{p}\right)
\end{aligned}
$$

sendo $\mathrm{P}_{0}$ a precipitação observada; $\mathrm{P}_{\mathrm{p}}$ a precipitação prevista; $\sigma$ o operador desvio padrão; $<>0$ operador de mediação e $\rho$ a correlação entre $\mathrm{P}_{0}$ e $\mathrm{P}_{\mathrm{p}}$.

\subsection{Esquema numérico de terceira-ordem corrente acima (ENTOCA)}

Os esquemas numéricos corrente acima têm aplicação em problemas de mecânica dos fluídos. Fisicamente, a curva característica que passa por um determinado instante de tempo e ponto no espaço dentro do domínio de integração é determinada por informações corrente acima do ponto. Logo, a característica pode ser conhecida a priori.

Os esquemas de primeira ordem amortecem o sinal, por exemplo, o esquema upstream (Mesinger,1973) promove o amortecimento seletivo de ondas muito curtas, porém os esquemas de maior ordem amortecem as ondas de forma ainda mais seletiva (Haltiner e Willians, 1980) seletivamente. Neste estudo, é apresentado o esquema de terceira-ordem derivado por Tremback et al. (1987), que emprega uma interpolação polinomial lagrangiana. O polinômio é usado com o objetivo de prever o campo de uma dada variável (neste caso a taxa de precipitação) para os intervalos de tempo futuros, por interpolação. De acordo com Pereira Filho et al. (2002), o ENTOCA resulta na seguinte expressão:

$$
\begin{gathered}
\phi_{j}^{n+1}=\phi_{j}^{n}+\frac{\sigma}{6}\left(-\phi_{j-2}^{n}+6 \phi_{j-1}^{n}-3 \phi_{j}^{n}-2 \phi_{j+1}^{n}\right) \\
+\frac{\sigma^{2}}{2}\left(\phi_{j-1}^{n}-2 \phi_{j}^{n}+\phi_{j+1}^{n}\right)+\frac{\sigma^{3}}{2}\left(\phi_{j-2}^{n}-3 \phi_{j-1}^{n}+3 \phi_{j}^{n}-\phi_{j+1}^{n}\right)
\end{gathered}
$$

sendo $\phi$ (campo escalar a ser advectado), $n$ o nível de tempo, $j$ o índice do ponto de grade, $\sigma=U \Delta \mathrm{t} / \Delta \mathrm{x}$ o número de Courant, $U$ velocidade do vento, constante e positivo, $\Delta t$ o passo de tempo da advecção e $\Delta x$ a resolução da grade. A Equação 8 pode ser escrita de forma mais compacta,

$$
\phi_{j}^{n+1}=\phi_{j}^{n}+\sum_{i=1}^{3} \sigma_{i}\left(\vec{C}_{i} \cdot \vec{P}_{j}\right)
$$

com $\sigma_{1}=\sigma_{2}=\sigma_{3}=\sigma / 6, C_{1}=(-1,6 ;-3 ;-2), C_{2}=(0 ; 1$; $-2,1), \mathrm{C}_{3}=(1 ;-3,3 ;-1)$ e $P_{j}=\left(\phi_{j-2}^{n}, \phi_{j-2}^{n-1}, \phi_{j-2}^{n+1}\right)$. Quando as componentes do vento (u ou v) são negativas, as entidades vetoriais da advecção lagrangiana são reescritas, com as derivadas calculadas sempre de acordo com acordo com a direção a barlavento (upwind), como $\mathrm{C}_{1}=(1 ;-6,3 ; 2), \mathrm{C}_{2}=(-1,3$; $-3 ; 1), \mathrm{C}_{3}=(1 ;-3,3 ;-1)$ e $P_{j}=\left(\phi_{j+2}^{n}, \phi_{j+1}^{n}, \phi_{j}^{n}, \phi_{j-1}^{n}\right)$. A mudança direcional da estimativa das derivadas produz uma imagem

Tabela 1 - Contagem das previsões de curtíssimo prazo com resolução de $2 \mathrm{~km}$ para um determinado limite de chuva horária (Po) contra os respectivos valores observados com o RSP e fórmulas de obtenção dos índices CSI, POD e FAR.

\begin{tabular}{|c|c|c|}
\hline Desempenho & Observação P > P0 & Observação $\mathbf{P}<\mathbf{P 0}$ \\
\hline Previsão P > P0 & Acerto & Falso Alarme \\
\hline Previsão P $<$ P0 & Erro & Acerto \\
\hline
\end{tabular}




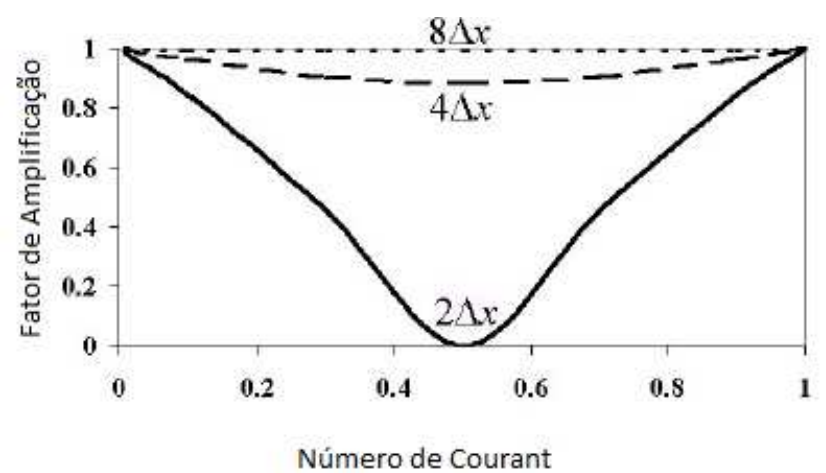

Figura 1- Esquema de amplificação de ondas curtas do esquema ENTOCA.

FONTE: Pereira Filho (2002).

especular da Equação 8, enquanto mantém invariante o vetor $\mathrm{C}_{2}$.

A análise de estabilidade de Von Neumann indica que: (a) o esquema é dominado por erros de fase (derivadas de ordem impar na equação modificada do erro de truncamento); (b) o critério de estabilidade é $\alpha \leq 1$. Os fatores de amplificação de ondas curtas até $8 \Delta \mathrm{x}$ são apresentados na Figura 1. Como já fora dito, o esquema numérico ENTOCA apresenta propriedades de amortecimento seletivo de ondas. O valor do número de Federich-Courant-Levy (CFL) de 0,5 permite remover completamente ondas com comprimento $2 \Delta x$. O intervalo de valores do CFL entre 0,3 e 0,7 implica amortecimento parcial das ondas curtas (Pereira Filho, 2002).

A assimetria do ENTOCA requer a determinação da direção corrente acima, o que requer mais processamento do que os esquemas de ordem par (Tremback, 1987). A integração bidimensional é realizada nas seguintes etapas:

$$
\begin{aligned}
& \text { (a) } \phi_{j, k}^{i}=\phi_{j, k}^{n}+\sum_{i=1}^{3} \sigma_{i}(u)\left(\vec{C}_{i} \cdot \vec{P}_{j}\right)_{k} \\
& \text { (b) } \phi_{j, k}^{n+1}=\phi_{j, k}^{i}+\sum_{i=1}^{3} \sigma_{i}(v)\left(\vec{C}_{i} \cdot \vec{P}_{k}\right)_{j}
\end{aligned}
$$

Este método de integração não altera o critério de estabilidade (Tremback, 1987) e considera o efeito das derivadas cruzadas na expansão de Taylor. Porém, se o vento é variável, pode haver uma fraca instabilidade do ENTOCA causada por derivadas espúrias (Smolarkiewicz, 1982).

\section{RESULTADOS}

A classificação dos sistemas precipitantes na área de cobertura do RSP foi realizada por meio da inspeção visual dos campos de taxa de precipitação. Os sistemas precipitantes foram classificados inicialmente quanto à organização dos ecos de chuva, distribuição horizontal, deslocamento e duração (ciclo de vida) possibilitando dessa forma, a identificação dos seguintes sistemas meteorológicos: Frentes Frias (FF), Bandas Dispersas de precipitação (BD), Linhas de Instabilidade (LI), Convecção Isolada (CI) e Brisa Marítima (BM). A frequência mensal dos diferentes sistemas precipitantes para o período de 2003 a 2007 a Leste de SP é apresentada na Figura 2.

Posteriormente, os sistemas foram classificados em convectivos e estratiformes, sendo o critério utilizado, baseado na intensidade da taxa de precipitação, conforme Santos da Silva et al. (2006) sendo que os sistemas com taxa de precipitação inferior a $20\left(\mathrm{~mm} \mathrm{~h}^{-1}\right)$ foram classificados como estratiformes, e sistemas com taxa de precipitação superior a $20\left(\mathrm{~mm} \mathrm{~h}^{-1}\right)$ como convectivos. Este limiar é arbitrário, outros poderiam ser usados, como por exemplo, uso de quartis para definir os limiares.

A frequência dos eventos estratiformes e convectivos é mostrada na Figura 3 para o período de 2003 a 2007. A precipitação convectiva apresenta alto gradiente horizontal

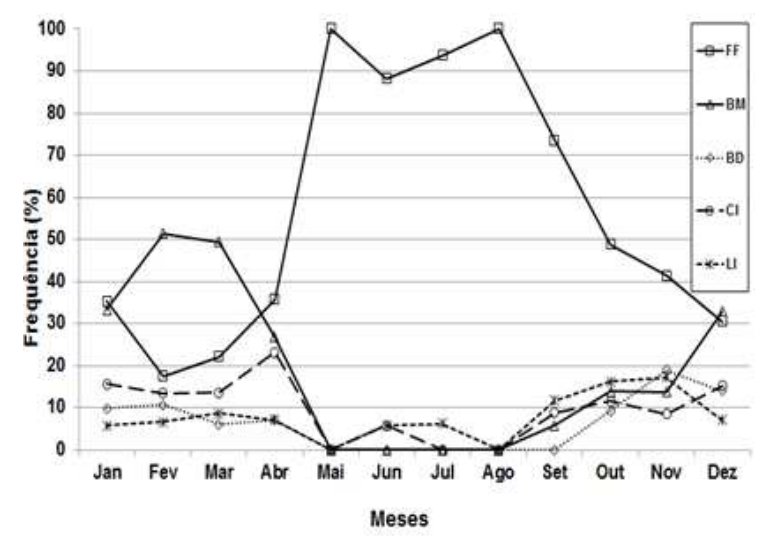

Figura 2- Frequência mensal dos diferentes sistemas precipitantes para o período de 2003 a 2007 a Leste de SP. Na legenda indica-se: Frentes frias (FF) (linha contínua com quadrados), Brisa marítima (BM) (linha contínua com triângulos), Banda Dispersa (BD) (linha pontilhada), Convecção Isolada (CI) (linha de traços longos) e Linhas de instabilidade (LI) (linha de traços curtos).

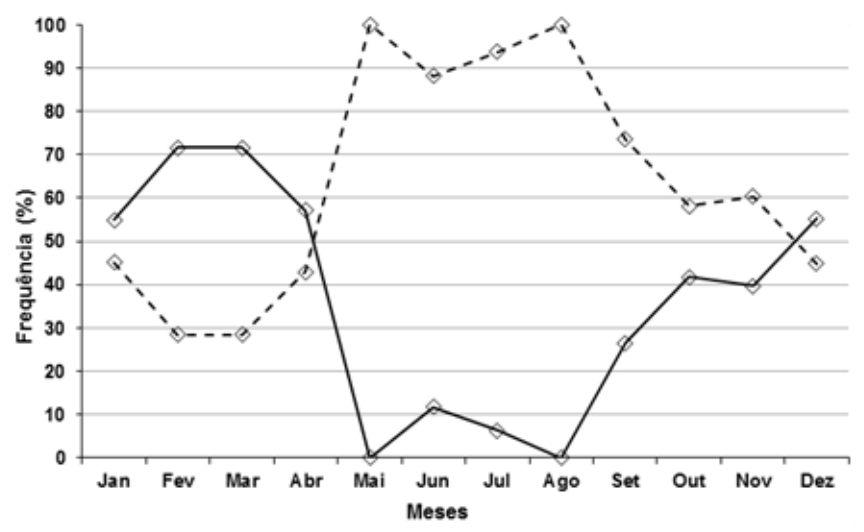

Figura 3- Frequências mensais de eventos estratiformes (linha tracejada) e convectivos (linha contínua) para o período de 2003 a 2007. 
de taxas de precipitação, taxas de precipitação elevadas e correntes ascendentes intensas. Por outro lado, baixas taxas de precipitação apresentam baixo gradiente horizontal de precipitação e correntes ascendentes fracas caracterizando a região de precipitação estratiforme (Chandrasekar e Zafar, 2004). Um limiar para a taxa de precipitação estratiforme igual a $20\left(\mathrm{~mm} \mathrm{~h}^{-1}\right)$ foi escolhido com base nos gradientes de precipitação e na climatologia dos sistemas precipitantes a leste de SP.

Os campos de precipitação mostrados a seguir (Figuras 4 a 8) são resultados da precipitação acumulada pelo RSP para $3 \mathrm{~h}$ (180 minutos) e da precipitação acumulada prevista pelo modelo advectivo para o mesmo intervalo de tempo. Os horários mostrados correspondem às simulações de 30, 60, 90, 120 e 180 minutos. As figuras correspondentes ao horário de 150 minutos foram omitidas por serem semelhantes aos campos correspondentes às de 180 minutos. A análise dos campos acumulados pelo radar e previsto pelo modelo para os exemplos de sistemas precipitantes apresentados mostra que, em geral, há uma subestimação dos resultados do modelo advectivo em relação às observações com o radar. Isto é atribuído à limitação do modelo de previsão ao não representar a dinâmica dos sistemas durante a extrapolação dos ecos de radar.
A avaliação das previsões de chuva com o modelo advectivo aplicado aos diferentes tipos de sistemas precipitantes foi realizada por meio dos índices CSI, POD e FAR nos limiares de altura de chuva acumulada pré-determinados e iguais a 0,2 $\mathrm{mm}, 2 \mathrm{~mm}$ e $16 \mathrm{~mm}$. A Figura 9 mostra os valores dos índices estatísticos para as acumulações de 0,2 e $16 \mathrm{~mm}$. Os valores para a acumulação de $2 \mathrm{~mm}$ são intermediários aos obtidos para as acumulações de 0,2 e $16 \mathrm{~mm}$, e por isto não são mostrados aqui. O pior desempenho da previsão de chuva para cada tipo de sistema precipitante foi apresentado para o limiar de 16,0 mm de chuva acumulada.

Os resultados mostram que quanto maior o limiar de chuva acumulada, mais difícil se torna a previsão devido à natureza variável dos sistemas precipitantes e o papel mais importante de outros mecanismos dinâmicos e termodinâmicos do sistema precipitante frente à advecção horizontal. O melhor desempenho do modelo advectivo foi obtido para previsões de FFs com CSI de $77 \%$. O desempenho máximo do modelo para 60 minutos de previsão de chuva acumulada para LIs, BDs, CIs e BMs são 67,5\%; $58 \% ; 56,4 \%$ e $47 \%$, respectivamente. Estes resultados são compatíveis com os de Pereira Filho et al. (1999), para uma resolução espacial de $2 \mathrm{~km} \times 2 \mathrm{~km}$.

RADAR: $30 \mathrm{~min}$

Figura 4- Campos de precipitação acumulados para 30 e 60 minutos observados pelo radar (coluna esquerda) e previstos pelo modelo advectivo (coluna direita), associados ao evento de Brisa Marítima do dia 02/jan/2005 às 17:32 (UTC). Latitudes, longitudes e contornos geográficos incluindo RMSP estão indicados. As escalas de cores indicam a precipitação acumulada em (mm). 


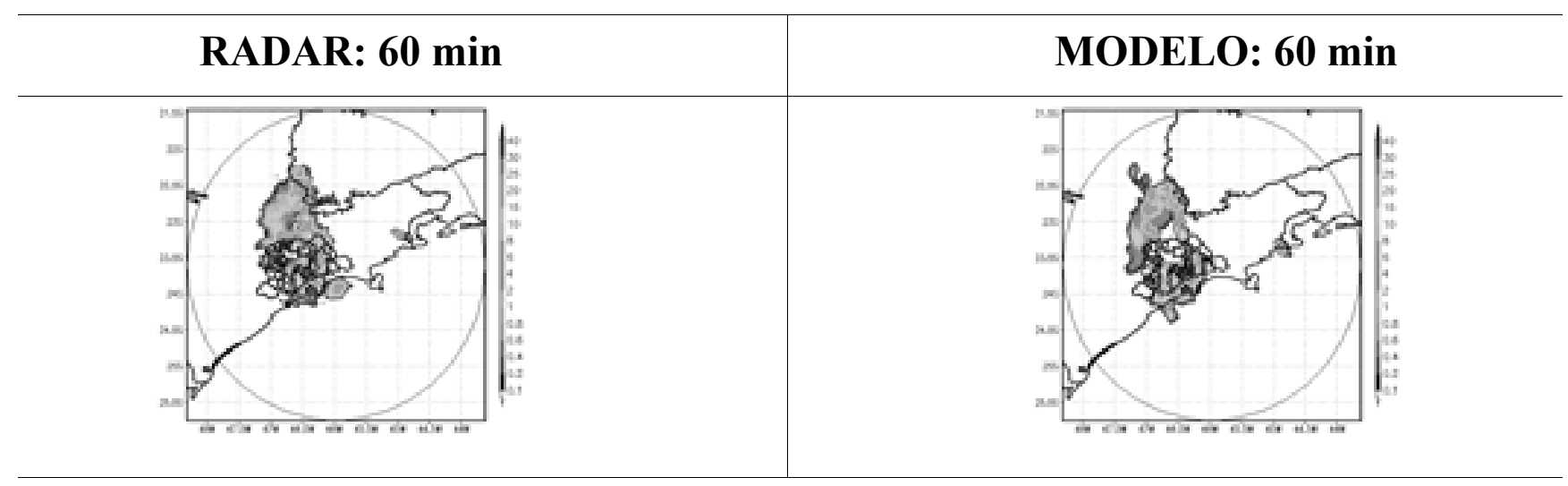

Figura 5- Idem à Figura 4, para Linha de Instabilidade do dia 19/set/2004 às 20:47 (UTC) para 60 minutos de acumulação.

RADAR: $60 \mathrm{~min}$ MODELO: $60 \mathrm{~min}$

Figura 6- Idem à Figura 4, para Sistema Frontal do dia 19/jun/2005 às 00:10 (UTC) para 60 minutos de acumulação.

RADAR: $60 \mathrm{~min}$

Figura 7- Idem à Figura 4, para Convecção Isolada do dia 18/abr/2004 às 17:12 (UTC) para 60 minutos de acumulação.

O campo do coeficiente de correlação linear (Figura 10) permitiu correlacionar o comportamento dos diferentes tipos de sistemas precipitantes ao longo do processo de integração no tempo. Os sistemas mais organizados apresentaram o maior grau de correlação linear da previsão, em torno de $75 \%$ para 120 minutos de advecção. Menor grau de correlação foi encontrado para os sistemas menos organizados, altamente variáveis, tanto no tempo, quanto no espaço.
Os erros das previsões em relação às observações com o RSP para os diferentes sistemas precipitantes, que atuam na RMSP, foram analisados por meio do EQM e dos seus componentes de amplitude e fase. A acurácia da previsão para até 3h de precipitação acumulada em termos do EQM é apresentada na Figura 11. Os erros diminuem dos sistemas convectivos para os estratiformes. Esse gráfico quantifica os erros das previsões de chuva com o modelo advectivo. 


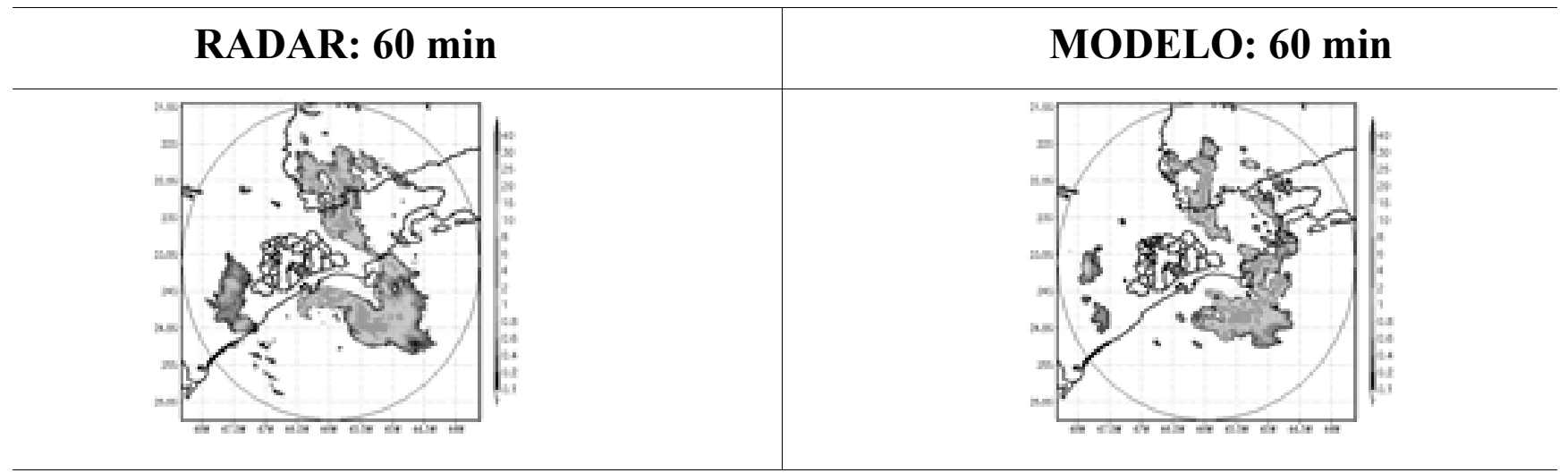

Figura 8- Idem à Figura 4, para Banda Dispersa do dia 21/abr/2005 às 07:47 (UTC) para 60 minutos de acumulação.

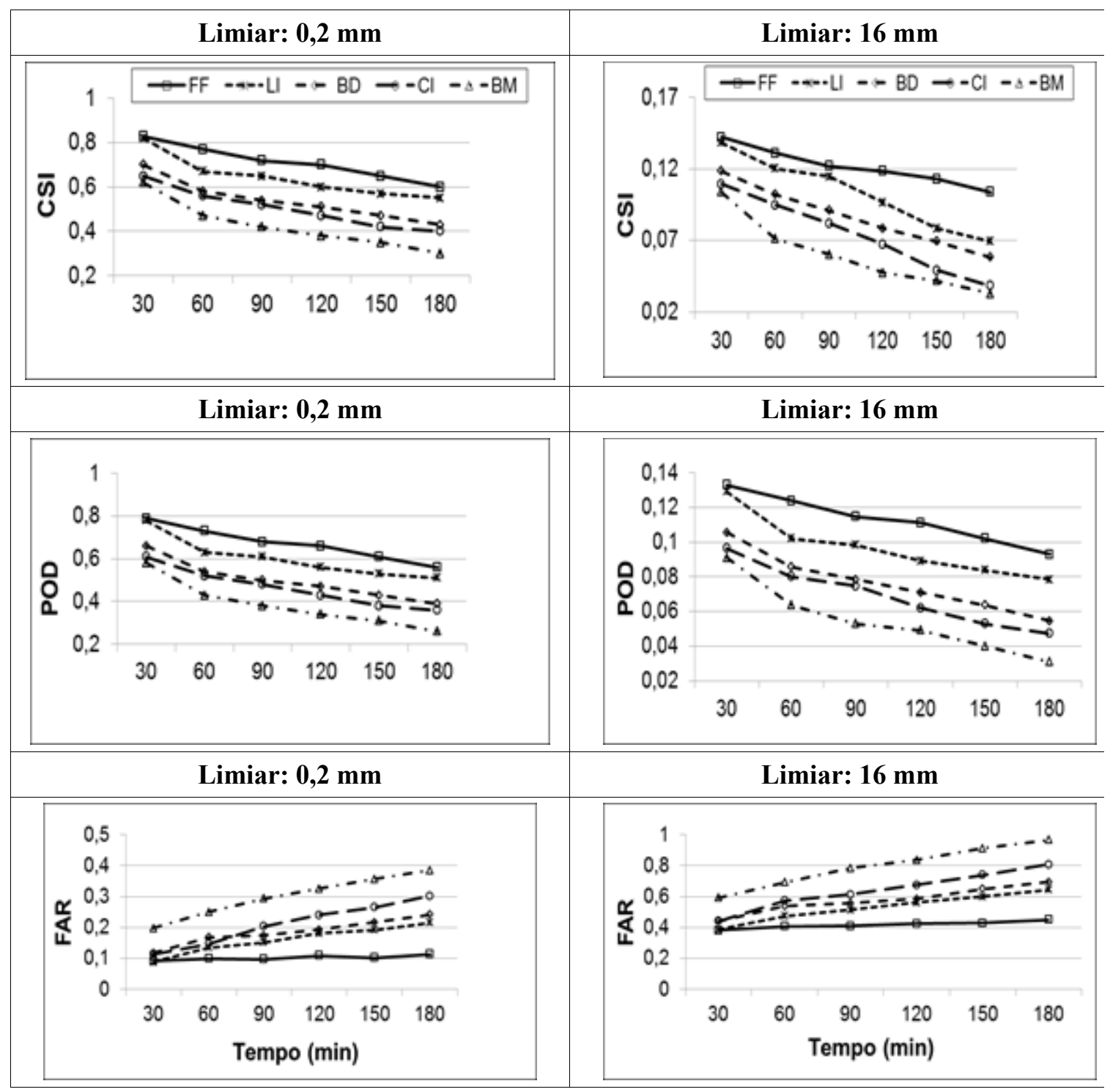

Figura 9- Desempenho das previsões de chuva a curtíssimo prazo para os diferentes sistemas precipitantes, pelos índices CSI, POD e FAR, mostrados para os limiares de chuva acumulada de $0,2 \mathrm{~mm}$ (coluna esquerda) e $16 \mathrm{~mm}$ (coluna direita). 


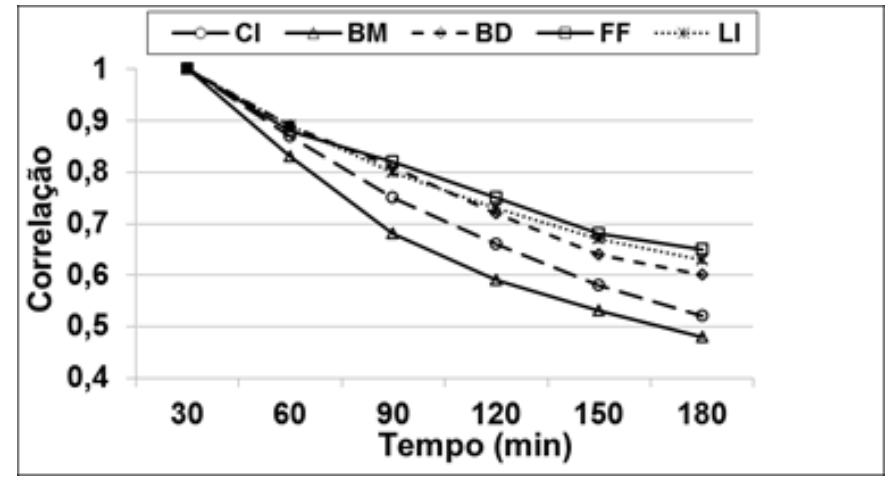

Figura 10 - Coeficiente de correlação da previsão para os diferentes tipos de sistemas precipitantes como monitorados pelo RSP.

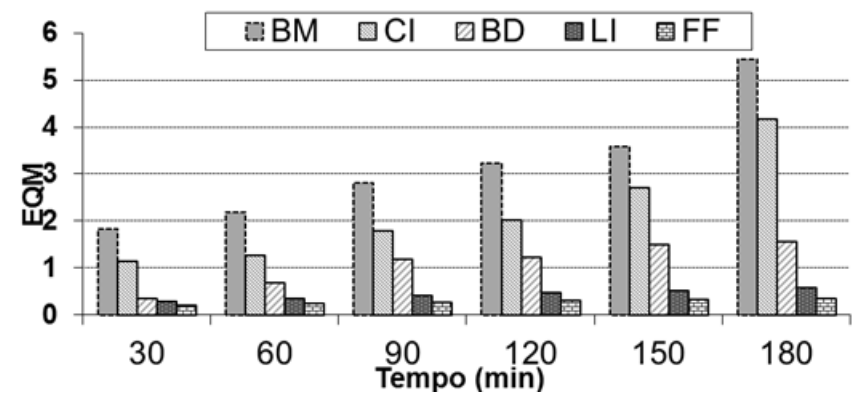

Figura 11 - Erro Quadrático Médio da precipitação acumulada em 3 horas e prevista pelo modelo advectivo em relação ao estimado com o RSP.
A Figura 12a mostra a análise do termo de amplitude do EQM. Este termo indica os erros na quantificação da chuva pelo modelo advectivo para cada tipo de sistema. Os maiores valores no erro da amplitude estão relacionados aos sistemas convectivos e os menores, aos estratiformes. Finalmente, a Figura $12 \mathrm{~b}$ apresenta a análise do termo de fase do EQM. Este termo está relacionado à posição do sistema. Os maiores erros na detecção da posição dos sistemas estão associados aos sistemas convectivos e os menores, aos estratiformes.

\section{CONCLUSÕES}

Os resultados obtidos com o modelo advectivo apresentaram melhor desempenho das mais baixas para as mais altas acumulações de precipitação. Assim como, para os sistemas com estrutura da precipitação mais organizada, como linhas de instabilidade, ou estratiforme, como sistemas frontais (em rápida movimentação, quando a advecção é horizontal o principal mecanismo).

A correlação linear mostrou que os eventos estratiformes também possuem o maior grau de correlação, decorrência do grau de organização dos sistemas e do ciclo de vida dos mesmos, fatores determinantes no limite da previsibilidade destes, para uma previsão de até 3 horas de chuva acumulada.

As menores incertezas das previsões de chuva com o modelo advectivo estão relacionadas com os sistemas precipitantes que possuem uma estrutura morfológica mais homogênea e mais organizada da precipitação, assim como um

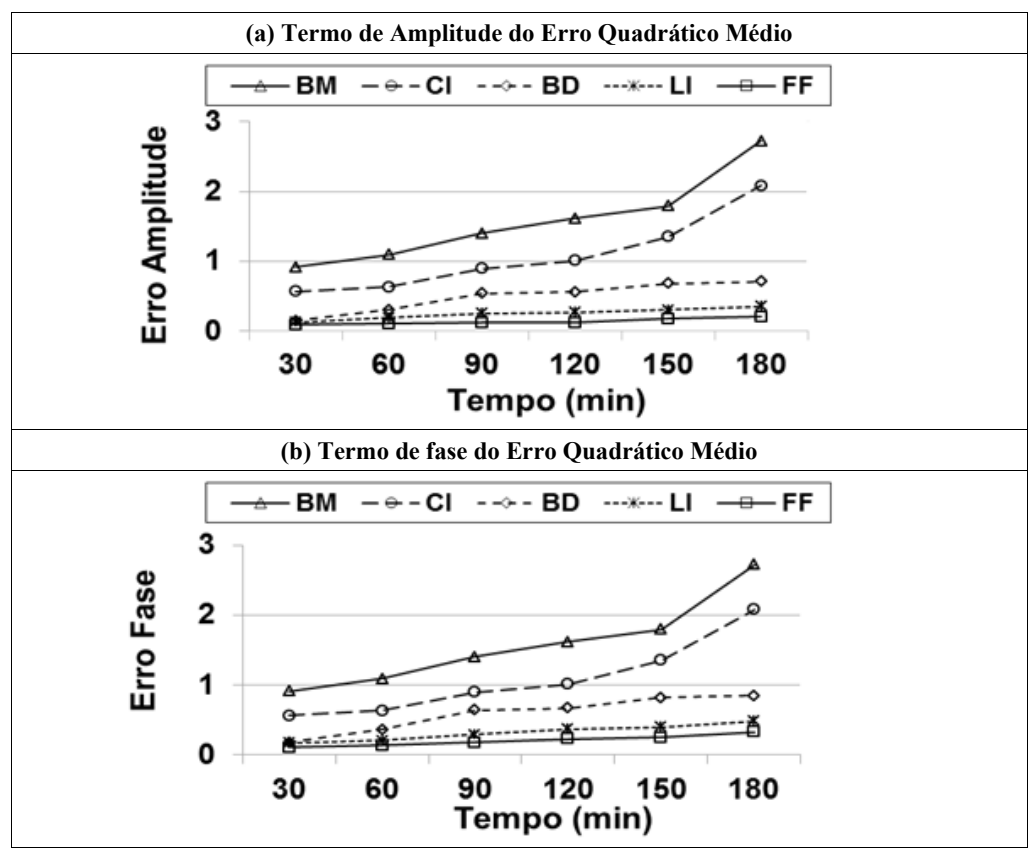

Figura 12 - Termo de amplitude do Erro Quadrático Médio da precipitação acumulada em 3 horas prevista pelo modelo advectivo em relação ao estimado pelo RSP (a) e termo de fase do Erro Quadrático Médio (b). 
ciclo de vida maior. Isto permite que estes sistemas possam ser advectados por um período superior a 90 minutos. Nos sistemas convectivos que apresentam maiores incertezas, a previsão mais acurada foi obtida para intervalos de tempo acima de 30 minutos nos casos de situação convectiva e entre 60 e 90 minutos, para os casos de convecção mais organizada.

A amplitude do EQM apresentou maior erro comparado à fase. A análise dos resultados permite classificar a ordem de acurácia da previsão de chuva a curtíssimo prazo na RMSP com o modelo advectivo, na seguinte ordem decrescente: Frentes Frias, Linhas de Instabilidade, Bandas Dispersas, Convecção Isolada e Brisa Marítima, respectivamente.

Como sugestões para trabalhos futuros as seguintes questões são propostas:

1) incluir um maior número de casos para avaliação da previsão,

2) avaliar o desempenho da previsão a curtíssimo prazo para cada estação do ano,

3) comparar a metodologia proposta contra modelos hidrológicos para estudos na RMSP, e

4) incluir outros efeitos como estágio de desenvolvimento precipitante no algoritmo.

\section{AGRADECIMENTOS}

Os autores agradecem à Fundação de Amparo à Pesquisa do Estado de São Paulo (FAPESP) pelo apoio ao projeto de pesquisa e pela bolsa (projeto número 06/58516-9) e ao Dr. Ricardo Hallak, pelo apoio e auxílio em muitas etapas deste trabalho.

\section{REFERÊNCIAS BIBLIOGRÁFICAS}

AUSTIN, G.L. E BELLON. The use of digital radar records for short-term precipitation. Quarterly Journal of the Royal Meteorological Society, v.100, p.658-664, 1974.

BELLON, A. E AUSTIN, G.L. The evaluation of two years of real-time operation of a short term precipitation forecasting procedure (SHARP). Journal of Applied Meteorology, v. 17, p. 1778-1787, 1978.

BELLON, A.; AUSTIN, G. The accuracy of short-term radar rainfall forecasts. Journal of Hydrology, v. 70, p. 35-49, 1984.

BRINGI, V.N; CHANDRASEKHAR, V. Polarimetric DopplerWeather Radar. Principles and Applications. Cambridge University Press, NY, Sep 8, p. 664, 2005.

BROWNING, K.A. E COLLIER, C.G. Nowcasting of precipitation systems. Reviews of Geophysics, v. 27, p. 345-370, 1989.

BROWNING, K.A.; COLLIER, C.G.; LARKE, P.R.; MENMUIR, P.; MONK, G.A.; OWENS, R.G. On the forecasting of frontal rain using a weather radar network. Water Resources Research, v. 110, p. 534-552, 1982.

CHANDRASEKAR, V. E ZAFAR, B. Precipitation Type Determination From Spaceborne Radar observations. IEEE Transactions on Geoscience and Remote Sensing, v. 42, p. 10, p.2248-2253, 2004.

EINFALT, T.; DENOLUX, T.; JACQUET, G. A radar rainfall forecasting method designed for hydrological purposes. Journal of Hydrology, v. 114, p. 229-244, 1990.

ELVANDER, R.C. An evaluation of the relative performance of three weather echo forecasting techniques. In: Preprint v. 526-532 of the 17th CONFERENCE. ON RADAR METEOROLOGY, Seattle. AMS, Boston, 1976.

GAO, S.; LI, X. Cloud-resolving modeling of convective processes - New York, NY: Springe, p.219, 2010.

GEORGAKAKOS, K.P.; BRAS, R.L. A hydrologically useful station precipitation model I. Formulation. Water Resources Research, v. 20, p. 1585-1596, 1984a.

GEORGAKAKOS, K.P.; BRAS, R.L. A hydrologically useful station precipitation model 2. Case studies. Water Resources Research, v. 20, p. 1597-1610, 1984 b.

HALTINER, G. J.; WILliANS, R. T. Numerical Prediction and Dynamic Meteorology. New York, John Wiley and Sons, p.477, 1980.

JOHNSON, E.R.; BRAS, R.L. Multivariate short-term rainfall prediction. Water Resources Research, v. 16,p. 173-185, 1980.

MESINGER, F. A method for construction of second-order accurate difference schemes permitting no false two-gridinterval waves in the height field. Tellus, v. 25, p. 444-457, 1973.

NOVÁK, P. The Czech Hydrometeorological Institute's Severe Storm Nowcasting System. Atmospheric Research, v.83, p. 450-457, 2007.

PEREIRA FILHO, A.J. Uso do radar meteorológico para previsão de chuva a curto-prazo aplicado à previsão hidrológica em bacias urbanizadas. Tese de mestrado, Departamento de Ciências Atmosféricas, Universidade de São Paulo, IAG/USP, p. 150, 1989.

PEREIRA FILHO, A.J.; CRAWFORD, K.C.; STENSRUD, D.J. Mesoscale precipitation fields: part II: hydrometeor logic modeling, Journal of Applied Meteorology, v. 38, n.1, p. 101-125, 1999.

PEREIRA FILHO, A.J. The positive definite and the thirdorder upstream schemes applied to $2 \mathrm{D}$ frontogenesis problem: intercomparison and optimization. $2^{\circ}$ SIMPÓSIO BRASILEIRO DE MODELAGEM ATMOSFÉRICA DO XII CONGRESSO BRASILEIRO DE METEOROLOGIA, Foz do Iguaçu, PR, 2002, 1 CD-ROM.

SÁLEK M.; BREZKOVÁ L.; NOVÁK P. The use of radar in hydrological modelling in the Czech Republic - case 
studies of flash floods. Natural Hazards and Earth System Sciences, v. 6, p. 229-236, 2006.

SANTOS DA SILVA, F.D. Análise Objetiva estatística da precipitação estimada com radar e medida por uma rede telemétrica. Dissertação de mestrado, Departamento de Ciências Atmosféricas, Universidade de São Paulo, IAG/ USP. 2006, 101 p.

SMOLARKIEWICZ, P.K. The multidimensional Crowley advection scheme. Monthly Weather Review, v. 11, p. 1968-1983, 1982.

TAKACS, L.L. A two-step scheme for the advection equation with minimized dissipation and dispersion errors. Monthly Weather Review, v. 113, p. 1050-1065, 1985.

TREMBACK, C. J.; POWELL, J.; COTTON, W. R.; PIELKE, R. A. The forward-in-time upstream advection scheme: extension to higher orders. Monthly Weather Review, v. 115, p. 540-555, 1987.
WALTON, M.; JOHNSON, E. An improved precipitation projection procedure for the NEXRAD Flash-Flood Potential system. Preprints, 23rd Conf. Radar Meteorology, American Meteorological Society, JP62-JP65, 1986.

WILKS, D.S. Statistical Methods in the Atmospheric Sciences: An Introduction. Academic Press, p. 467, 1995.

WILSON, J. W.; CROOK, N. A.; MUELLER, C. K.; SUN, J.; DIXON, M. Nowcasting Thunderstorms: A Status Report, Bulletin of the American Meteorological Society, v. 79, p. 2079-2099, 1998. 